\title{
Prevalence and Prognostic Value of Psychological Stress Events in Patients with First Myocardial Infarction-Long-Term Follow-Up Study
}

\author{
Andrea Jaensch ${ }^{1}$, Ben Schöttker 2,3 ${ }^{\mathbb{D}}$, Roman Schmucker ${ }^{4}$, Wolfgang Koenig 1,5,6, Hermann Brenner ${ }^{2,3} \mathbb{D}$ \\ and Dietrich Rothenbacher $1,2, *$ (D) \\ 1 Institute of Epidemiology and Medical Biometry, Ulm University, 89081 Ulm, Germany; \\ andrea.jaensch@uni-ulm.de (A.J.); koenig@dhm.mhn.de (W.K.) \\ 2 Division of Clinical Epidemiology and Ageing Research, German Cancer Research Center (DKFZ), \\ 69120 Heidelberg, Germany; b.schoettker@Dkfz-Heidelberg.de (B.S.); h.brenner@Dkfz-Heidelberg.de (H.B.) \\ 3 Network Ageing Research, University of Heidelberg, 69115 Heidelberg, Germany \\ 4 Klinik Schwabenland, 88316 Isny-Neutrauchburg, Germany; Roman.Schmucker@wz-kliniken.de \\ 5 Deutsches Herzzentrum München, Technische Universität München, 80636 Munich, Germany \\ 6 German Centre for Cardiovascular Research (DZHK), Partner Site Munich Heart Alliance, \\ 80636 Munich, Germany \\ * Correspondence: dietrich.rothenbacher@uni-ulm.de; Tel.: +49-(0)731/50-31060
}

check for

updates

Citation: Jaensch, A.; Schöttker, B.; Schmucker, R.; Koenig, W.; Brenner, H.; Rothenbacher, D. Prevalence and Prognostic Value of Psychological Stress Events in Patients with First Myocardial Infarction-Long-Term Follow-Up Study. J. Clin. Med. 2021, 10, 3562. https://doi.org/10.3390/ jcm10163562

Academic Editor: Salvatore De Rosa

Received: 9 July 2021

Accepted: 11 August 2021

Published: 13 August 2021

Publisher's Note: MDPI stays neutral with regard to jurisdictional claims in published maps and institutional affiliations.

Copyright: (c) 2021 by the authors. Licensee MDPI, Basel, Switzerland. This article is an open access article distributed under the terms and conditions of the Creative Commons Attribution (CC BY) license (https:/ / creativecommons.org/licenses/by/ $4.0 /)$.

\begin{abstract}
While there is good evidence that symptoms of depression determine prognosis of patients with coronary heart disease (CHD), the role of psychological stress is less clear. We evaluated the prognostic value of stressful events in patients with initial myocardial infarction (MI) with respect to subsequent cardiovascular events. The KAROLA-study included patients with CHD who participated in an in-patient rehabilitation program. A total of 577 patients with initial MI were included and self-reported psychological stressful events before their MI was assessed by a structured questionnaire. Hazard ratios were used to evaluate the long-term association of stressful events with secondary cardiovascular events. Additionally, associations of stressful events with depression, anxiety and other cardiovascular risk factors were investigated. Unusual stress at work (26.5\%) and sleep disorder $(23.4 \%$ ) were the most frequently reported stressful events that occurred in the last 4 weeks before MI. However, only death of a family member showed a statistically significant increase in risk for subsequent cardiovascular events (HR: 1.59; 95\%-CI: 1.01-2.50) and this result was not corrected for multiple testing. Notably, we found higher symptom scores of anxiety and depression associated with all single stressful event items. In conclusion, we found no clear patterns that psychological stressful events before MI would increase the long-term risk of subsequent adverse CHD events directly. However, we saw increased symptom scores of anxiety and depression in persons with stressful events.
\end{abstract}

Keywords: coronary heart disease; myocardial infarction; psychological stress events; depression; anxiety

\section{Introduction}

Patients with coronary heart disease (CHD) often report symptoms of depression and anxiety [1,2]. There is good evidence that especially symptoms of depression determine the prognosis of patients with CHD [3]. As a consequence, a higher priority is given to psychosocial factors in recent cardiologic guidelines, and mental health risk factors such as depression are established risk and prognostic factors for CHD. Yet they seem to be under-recognized in their importance, and are often not incorporated in secondary prevention strategies in clinical practice, especially during follow-up care [4].

However, besides mood disorders such as depression and anxiety, other negative psychological stress factors receive less attention, and evidence from studies is scarce. Little 
data exists about the prevalence and the role of psychological stress and trauma as triggers of acute events and even less about their role as prognostic factors for long-term outcomes. These psychological risk factors and psychological stress are probably also associated with established cardiovascular risk factors and associated adverse endpoints. However, key questions about directionality and possible causal relationships are still open [5].

The objective of this study was to evaluate the prevalence and the prognostic value of psychological stress events in patients with first myocardial infarction (MI) with respect to subsequent cardiovascular events.

\section{Materials and Methods}

\subsection{Study Population and Design}

KAROLA, a prospective cohort study, included all patients with incident CHD (International Classification of Diseases, 9th Rev. pos. 410-414) who participated in an in-patient rehabilitation program between January 1999 and May 2000 in one of two rehabilitation clinics in Germany (Schwabenland-Klinik, Isny, and Klinik am Südpark, Bad Nauheim). The age of the patients included was between 30 and 70 years (details of the overall CHD cohort in [3]). In Germany, all patients with acute MI or coronary artery revascularization (coronary artery bypass grafting (CABG), or percutaneous coronary interventions (PCI)) are offered an in-hospital rehabilitation program after discharge from the acute care hospital. This program lasts about three weeks and in KAROLA, only patients who were admitted within three weeks after their first acute event were included. During the recruitment period, $58 \%(n=1206)$ of all eligible patients agreed to participate. The study was approved by the Ethics Boards of the Universities of Ulm (No. 186/98) and Heidelberg and of the Physicians' chamber of the States of Baden-Württemberg and Hessen.

\subsection{Data Collection and Assessment of Psychological Stress Events}

All patients completed a standardized questionnaire at the beginning of the in-patient rehabilitation program, containing sociodemographic information, medical history and the German version of the Hospital Anxiety and Depression Scale (HADS) [6,7]. Patients after MI also received a structured questionnaire with further information on the MI event and its circumstances. Only these patients were included in this study $(n=577)$. Psychological stress events were asked as follows: "Did one of the following events happen in the time before infarction, which was very stressful for you?". Possible answers were "No", "Yes, within $24 \mathrm{~h}$ before infarction", "Yes, within 4 weeks before infarction" and "Yes, more than 4 weeks before infarction". The following events were recorded one after another: "Death of family member", "Death of friend", "Personal disease", "Unusual stress at work", "Mental excitement/dispute", "Sleep disorders", "Changes at workplace" and "Changes in partner relationship". Besides evaluating the single items, a score-variable was also built by summing up the events.

\subsection{Follow-Up and Evaluation of Cardiovascular Disease Events}

For follow-up, all patients and their primary care physicians were contacted simultaneously by mail one, three, four and a half, six, eight, ten and fifteen years after discharge from in-patient rehabilitation. Patients completed a standardized questionnaire and primary care physicians were asked about adverse cardiovascular disease events and treatment since discharge from the rehabilitation clinic. In case of death during follow-up, the death certificate, with the date and main cause of death, was obtained from local Public Health departments. The main cause of death was coded according to the International Classification of Disease. A subsequent adverse cardiovascular disease event (CVD) was defined as: CVD as main cause of death (ICD-9 pos. 390-459; ICD-10 pos. I0-I99 and R57.0) or diagnosed non-fatal MI, or ischemic cerebrovascular event (TIA or stroke) as reported by the primary care physicians. 


\subsection{Statistical Analysis}

After describing the study population with respect to various sociodemographic and medical characteristics, the prevalence of stressful events occurring within 4 weeks before MI was determined. To investigate the association of stressful events with subsequent non-fatal and fatal secondary CVD events, we fitted a Cox proportional hazard model for 15 years of follow-up (hazard ratio (HR) and its $95 \%$ confidence interval (CI)). We also considered six years of follow-up, assuming that the association might be stronger during shorter follow-up periods, as the influence of specific point-in-time related stressful events might decrease over time. The basic model was adjusted for age and sex. Additional established potential confounders were considered in the second model: body mass index (BMI), education ( $<10$ years vs. $\geq 10$ years), rehabilitation clinic, smoking status (never, current, former-smoker), history of diabetes mellitus, left ventricular function (no or only little impairment, modest or severe impairment) and high-density lipoprotein cholesterol.

We also investigated the association of psychological stressful events with suspected cardiovascular risk factors such as body mass index (BMI), diastolic and systolic resting blood pressure, lipid parameters, cotinine level in serum, C-reactive protein, NT-proBNP, hs-cTnT, and symptom scores of depression and anxiety. Age- and sex-adjusted mean values were calculated and compared according to the presence or absence of each stressful event by means of Generalized Linear Regression Models. All statistical procedures were performed with the SAS statistical software package (release 9.4 SAS Institute Inc., Cary, NC, USA).

\section{Results}

The mean age of the 577 included patients with MI was 57.3 years at baseline and $84 \%$ of them were male. Further characteristics are displayed in Table 1.

Table 1. Descriptive table of the study population with myocardial infarction at baseline $(n=577)$.

\begin{tabular}{|c|c|}
\hline \multicolumn{2}{|l|}{ Characteristics at Baseline } \\
\hline Age (years) $\left(\mu, S^{\#}\right)$ & $57.3(8.6)$ \\
\hline Men, $n(\%)$ & $485(84.1 \%)$ \\
\hline Length of follow-up, years * & $13.0(6.7 ; 14.8)$ \\
\hline School education $<10$ years, $n(\%)$ & $342(59.3 \%)$ \\
\hline Body mass index $\left(\mathrm{kg} / \mathrm{m}^{2}\right),(\mu, \mathrm{SD})$ & $27.1(3.3)$ \\
\hline \multicolumn{2}{|l|}{ Smoking status, $n(\%)$} \\
\hline Never & $177(30.7 \%)$ \\
\hline Former & $365(63.3 \%)$ \\
\hline Current & $35(6.1 \%)$ \\
\hline History of diabetes, $n(\%)$ & $91(15.8 \%)$ \\
\hline History of hypertension, $n(\%)$ & $293(51.2 \%)$ \\
\hline History of heart failure, $n(\%)$ & $46(8.3 \%)$ \\
\hline \multicolumn{2}{|l|}{ Clinical score (angiographic evaluation), $n$ (\%) } \\
\hline 1 vessel disease & $209(36.2 \%)$ \\
\hline 2 vessel disease & $165(28.6 \%)$ \\
\hline 3 vessel disease & $152(26.3 \%)$ \\
\hline Unknown & $37(6.4 \%)$ \\
\hline \multicolumn{2}{|l|}{ Left ventricular function, $n(\%)$} \\
\hline Unknown & $41(7.1 \%)$ \\
\hline Little/no & $392(67.9 \%)$ \\
\hline Modest/severe & $144(25.0 \%)$ \\
\hline Percutaneous coronary intervention, $n(\%)$ & $316(55.1 \%)$ \\
\hline Coronary artery bypass graft, $n(\%)$ & $106(18.5 \%)$ \\
\hline HDL-cholesterol $\S(\mathrm{mg} / \mathrm{dL})(\mu, \mathrm{SD})$ & $40.3(11.0)$ \\
\hline C-reactive protein $(\mathrm{mg} / \mathrm{L}) *$ & $2.35(0.97 ; 5.87)$ \\
\hline NT-proBNP (ng/L) * & $606.3(291.6 ; 1193.0)$ \\
\hline hs-Troponin T (ng/L)* & $12.60(8.19 ; 21.40)$ \\
\hline
\end{tabular}

* Median (Interquartile range). \# Standard deviation. § High-density lipoprotein cholesterol.

As depicted in Table 2, about one third of the patients reported unusual stress at work (33.0\%) or sleep disorders (28.0\%) before MI. Mental excitement or dispute (15.9\%) or personal disease $(14.7 \%)$ were also common. When only events within four weeks before 
MI were considered, unusual stress at work (26.5\%), sleep disorders $(23.4 \%)$, and mental excitement or dispute $(11.8 \%)$ remained the most commonly reported events.

Table 2. Number and percentage of persons with stressful events before myocardial infarction happened any time or within 4 weeks before infarction.

\begin{tabular}{ccc}
\hline & Any Time & Up to $\mathbf{4}$ Weeks Before Infarction \\
$\boldsymbol{n} \mathbf{( \% )}$ & $(\mathbf{\%})$
\end{tabular}

The risk for subsequent secondary CVD events $(n=171)$ during 15 years of follow-up increased for some of the stressful events, but none were statistically significant except death of a family member in the fully adjusted model (HR: 1.59; 95\%-CI: 1.01-2.50) (Table 3). In addition, a summary score of stressful events was not associated with adverse CVD outcomes in our multivariable model.

Table 3. Association of reporting stressful events before MI with fatal and non-fatal CVD events during 15 years of follow-up (FU).

\begin{tabular}{ccccc}
\hline & $n$ Events (\%) & $\begin{array}{c}\text { FU-Time in Years } \\
\text { (Median) }\end{array}$ & $\begin{array}{c}\text { HR (95\% CI) } \\
\text { Adjusted for Age and Sex }\end{array}$ & $\begin{array}{c}\text { HR (95\% CI) } \\
\text { Adjusted for Multiple } \\
\text { Covariates * }\end{array}$ \\
\hline Death of family member & $22(36.7 \%)$ & 12.2 & $1.44(0.92-2.26)$ & $1.59(1.01-2.50)$ \\
Death of friend & $9(33.3 \%)$ & 13.7 & $0.98(0.50-1.92)$ & $0.97(0.49-1.90)$ \\
Personal disease & $29(34.9 \%)$ & 13.0 & $1.22(0.82-1.82)$ & $1.20(0.80-1.79)$ \\
Unusual stress at work & $41(21.9 \%)$ & 13.8 & $0.69(0.48-1.00)$ & $0.77(0.53-1.12)$ \\
Mental excitement/dispute & $23(25.8 \%)$ & 13.1 & $0.97(0.62-1.51)$ & $0.98(0.62-1.53)$ \\
Sleep disorders & $51(32.1 \%)$ & 13.0 & $1.11(0.80-1.55)$ & $1.14(0.82-1.60)$ \\
Changes at workplace & $11(25.6 \%)$ & 13.0 & $1.14(0.61-2.13)$ & $1.19(0.63-2.24)$ \\
Changes in partner relationship & $7(36.8 \%)$ & 11.5 & $1.65(0.77-3.53)$ & $1.92(0.88-4.20)$ \\
Score of stressful events & $104(28.0 \%)$ & $13.0^{\#}$ & $1.02(0.90-1.16)$ & $1.05(0.92-1.20)$ \\
\hline
\end{tabular}

* Age, sex, BMI, education, rehabilitation clinic, smoking status, history of diabetes mellitus, left ventricular function and high-density lipoprotein cholesterol. " Score $>0$.

The association of stressful events before MI with age- and gender adjusted symptom scores of depression and anxiety and other established cardiovascular risk factors were evaluated at baseline. We found higher symptom scores of anxiety and depression associated with all single stressful event items. In detail, a statistically significant higher depression score was found among study participants who reported personal disease, temporarily unusual stress at work, sleep disorders, and changes in partner relationship. Symptoms of anxiety were significantly associated with personal disease, unusual stress at work, sleep disorders, changes at workplace and changes in partner relationship (Table 4). Most of the other well-established cardiovascular risk factors did not differ substantially between presence or absence of a specific stressful event and showed no consistent patterns with stressful events (Table S1 in the Supplementary Materials).

Table 4. Association of stressful events before MI with age- and sex-adjusted mean values of symptom scores of depression and anxiety.

\begin{tabular}{ccc}
\hline & Depression Score & Anxiety Score \\
\hline Death of family member & & \\
Yes & 5.65 & 6.74 \\
No & 4.78 & 5.99 \\
$p$-value & 0.089 & 0.18 \\
\hline
\end{tabular}


Table 4. Cont.

\begin{tabular}{|c|c|c|}
\hline & Depression Score & Anxiety Score \\
\hline \multicolumn{3}{|c|}{ Death of friend } \\
\hline Yes & 5.73 & 6.23 \\
\hline No & 4.85 & 6.08 \\
\hline$p$-value & 0.22 & 0.85 \\
\hline \multicolumn{3}{|c|}{ Personal disease } \\
\hline Yes & 5.67 & 7.10 \\
\hline No & 4.72 & 5.87 \\
\hline$p$-value & 0.033 & 0.010 \\
\hline \multicolumn{3}{|c|}{ Unusual stress at work } \\
\hline Yes & 5.33 & 6.88 \\
\hline No & 4.64 & 5.65 \\
\hline$p$-value & 0.059 & 0.002 \\
\hline \multicolumn{3}{|c|}{ Mental excitement/dispute } \\
\hline Yes & 5.32 & 6.75 \\
\hline No & 4.80 & 5.95 \\
\hline$p$-value & 0.24 & 0.088 \\
\hline \multicolumn{3}{|c|}{ Sleep disorder } \\
\hline Yes & 5.54 & 6.76 \\
\hline No & 4.48 & 5.66 \\
\hline$p$-value & 0.002 & 0.003 \\
\hline \multicolumn{3}{|c|}{ Changes at workplace } \\
\hline Yes & 5.81 & 7.46 \\
\hline No & 4.71 & 5.85 \\
\hline$p$-value & 0.074 & 0.016 \\
\hline \multicolumn{3}{|c|}{ Changes in partner relationship } \\
\hline Yes & 8.89 & 9.10 \\
\hline No & 4.69 & 5.92 \\
\hline$p$-value & $<0.001$ & 0.001 \\
\hline
\end{tabular}

\section{Discussion}

In this long-term cohort study including 577 patients with initial MI, besides an association of the stressful event "death of a family member", we found no consistent indication that psychologically stressful events before MI increased the long-term risk of subsequent adverse CHD events in a direct manner. However, we found an accumulation of increased symptom scores of anxiety and depression clustering in patients reporting stressful events. Nevertheless, the temporal sequence, especially regarding symptoms of anxiety and depression, the latter being recognized as a well-established prognostic risk factor, remains unclear. We also cannot discriminate causes from consequences of stress.

The prevalence of stressful events in our study varied considerably. Whereas changes in partner relationship were reported in only $3.4 \%$ of patients ( $0.5 \%$ during past four weeks), stress at work, with a prevalence of $33.0 \%$, and sleep disorders, with a prevalence of $28.0 \%$ (in past 4 weeks $26.5 \%$ and $23.4 \%$, respectively), were very common. Although there is much evidence that perceived work stress is associated with an increased risk of incident $\mathrm{CHD}$, evidence investigating the association of work stress with prognosis in patients with CHD is limited, but points to an increased risk for recurrent CVD events [8]. Kivimäki et al. [9] described that stress in adulthood may rather act as a trigger of acute CVD in high-risk populations for CVD, but there is also evidence that several stressors, such as perceived work stress, perceived stress and persistent psychological distress, are associated with adverse events in adults with existing CVD [9]. Work stress as measured by the well-established effort-reward imbalance instrument (i.e., high demand and low control or high effort and low reward) was associated with a $65 \%$ increase for recurrent CVD events in a meta-analysis done by Li et al. [8]. Perceived stress levels as measured by the validated perceived stress scale occurred with a $42 \%$ increased risk for 2-year mortality in a large cohort of patients with acute MI [10]. Although perceived stress was highly associated with depressive symptoms as in our study, the association of stress persisted after adjustment for depressive symptoms in their study. 
The evidence base for the association of anxiety and depression, especially for symptoms of depression, and their adverse relationship with prognosis in patients with CVD is better. It is likely that persistent psychological distress over time, instead of one-point measurements, should be considered. This was done in a study with the General Health Questionnaire over 4 years in 950 patients with a history of acute MI to demonstrate an association with long-term adverse outcomes [11].

In a previous analysis of the KAROLA-study, we had found a relevant prognostic association of anxiety and depression with further CHD events [3]. Stress at work and in family life, hostility, depression, anxiety and other mental disorders are considered as psychological risk factors in current ESC guidelines which contribute to the risk of developing incident CVD and also worsen the prognosis [4]. Screening is recommended at initial assessment and it appears that a long-term perspective should be implemented in the care of patients with CHD. Looking at the age- and sex-adjusted mean values, we clearly saw higher values of the anxiety and depression scores for patients with a stressful event. Furthermore, patients with one event were more likely to have other events. This indicates that a certain vulnerability or awareness of stress is more present in patients with a history of depression or anxiety. Psychological aspects such as purpose of life may also be important, as individuals with a high purpose of life may perceive stressors as less difficult, and might be less likely to react with unhealthy behaviour (i.e., unhealthy diet, smoking, less adherence to medication, etc.) [12].

The associations between stressful events and adverse cardiovascular events are complex. Interrelation with well-established behavioural and physiological risk factors such as smoking, unhealthy food choices, increased blood pressure, but also with symptoms of anxiety and depression, could all play a role. On the physiological levels, alterations in the autonomic nervous system and changes in other endocrine markers which are also related to hemostatic and pro-inflammatory changes, are discussed as possible links between stress, anxiety and depression and adverse cardiovascular outcomes, including death [4]. However, stressful events and other mental health disorders should be addressed and treated irrespective of a possible link with CHD prognosis.

This study has strengths and limitations. Overall, we investigated different domains of stressful events and investigated their association with established cardiovascular risk markers at baseline, and with mid-term as well as long-term adverse CVD events. Strengths include the long-term follow-up and the availability of well-established biomarkers. Furthermore, depression and anxiety were investigated with a standardized and validated questionnaire, which is established and worked well in patients with cardiac disease. Limitations include the lack of qualitative information to include additional sources of stressful events, the lack of validation of questions about psychological stressful events before MI and the one-time measurement. Moreover, as questions were asked after the events, recall and response bias might have been an issue. We did not correct for multiple testing (e.g., by means of Bonferroni-correction) and the study had a limited power due to the sample size. In addition, personality type and coping strategies were not assessed in patients with MI, as both might make an important difference. This notion is supported by the accumulation of stressful events within persons reporting such events and the association of stressful events with higher symptoms of anxiety and depression scores.

\section{Conclusions}

In conclusion, in patients with CHD, self-reported, different psychological stressful events before MI were not consistently associated with risk for subsequent CHD events. However, we found an accumulation of increased symptom scores of anxiety and depression clustering in patients reporting stressful events, suggesting that it might be useful to screen for mood disorders in patients with a history of stressful events during follow-up care and treat affected patients accordingly. 
Supplementary Materials: The following are available online at https:/ / www.mdpi.com/article / 10.3390/jcm10163562/s1, Table S1: Association of stressful events before MI with age- and sexadjusted mean values or concentrations (at the end of rehabilitation) of cardiovascular risk factors and biomarkers.

Author Contributions: D.R. and H.B. conceived the study. A.J. performed the statistical analysis. B.S., R.S., W.K. and H.B. participated in the design and conduct, and coordination of the study. A.J., B.S., R.S., W.K., H.B. and D.R. have been involved in drafting the manuscript or revising it critically for important intellectual content. All authors read and approved the final manuscript.

Funding: This research was funded by the Bundesministerium für Bildung und Forschung (grant \#01GD9820/0), the Pitzer Foundation (Bad Nauheim, Germany), and the Waldburg-Zeil Clinics (Isny, Germany).

Institutional Review Board Statement: The study was conducted according to the guidelines of the Declaration of Helsinki, and approved by the Ethics Committee of the University of Ulm (No. 186/98; date of approval: 3 November 1998).

Informed Consent Statement: Informed consent was obtained from all subjects involved in the study.

Data Availability Statement: The datasets used and/or analysed during the current study are available from the corresponding author on reasonable request.

Conflicts of Interest: Koenig reports personal fees from AstraZeneca, Novartis, Pfizer, The Medicines Company, DalCor, Kowa, Amgen, Corvidia, Daiichi-Sankyo, Berlin-Chemie, Genentech, OMEICOS, Esperion, Sanofi, Novo Nordisk, Bristol-Myers Squibb, grants and non-financial support from Beckmann, Singulex, Abbott, and Roche Diagnostics, outside the submitted work. The other authors report no COI.

\section{References}

1. Nicholson, A.; Kuper, H.; Hemingway, H. Depression as an aetiologic and prognostic factor in coronary heart disease: A meta-analysis of 6362 events among 146,538 participants in 54 observational studies. Eur. Heart J. 2006, 27, 2763-2774. [CrossRef]

2. Roest, A.M.; Martens, E.J.; Denollet, J.; de Jonge, P. Prognostic Association of Anxiety Post Myocardial Infarction with Mortality and New Cardiac Events: A Meta-Analysis. Psychosom. Med. 2010, 72, 563-569. [CrossRef] [PubMed]

3. Peter, R.S.; Meyer, M.L.; Mons, U.; Schöttker, B.; Keller, F.; Schmucker, R.; Koenig, W.; Brenner, H.; Rothenbacher, D. Long-term trajectories of anxiety and depression in patients with stable coronary heart disease and risk of subsequent cardiovascular events. Depress Anxiety 2020, 37, 784-792. [CrossRef] [PubMed]

4. Piepoli, M.F.; Hoes, A.W.; Agewall, S.; Albus, C.; Brotons, C.; Catapano, A.L.; Cooney, M.; Corrà, U.; Cosyns, B.; Deaton, C.; et al. 2016 European Guidelines on cardiovascular disease prevention in clinical practice. Eur. Heart J. 2016, 37, 2315-2381. [CrossRef]

5. Masters, K.S.; Shaffer, J.A.; Vagnini, K.M. The Impact of Psychological Functioning on Cardiovascular Disease. Curr. Atheroscler. Rep. 2020, 22, 51. [CrossRef]

6. Zigmond, A.S.; Snaith, R.P. The Hospital Anxiety and Depression Scale. Acta Psychiatr. Scand. 1983, 67, 361-370. [CrossRef]

7. Herrmann, C. International experiences with the Hospital Anxiety and Depression Scale-A review of validation data and clinical results. J. Psychosom. Res. 1997, 42, 17-41. [CrossRef]

8. Li, J.; Zhang, M.; Loerbroks, A.; Angerer, P.; Siegrist, J. Work stress and the risk of recurrent coronary heart disease events: A systematic review and meta-analysis. Int. J. Occup. Med. Environ. Health 2015, 28, 8-19. [CrossRef]

9. Kivimäki, M.; Steptoe, A. Effects of stress on the development and progression of cardiovascular disease. Nat. Rev. Cardiol. 2018, 15, 215-229. [CrossRef] [PubMed]

10. Arnold, S.V.; Smolderen, K.G.; Buchanan, D.M.; Li, Y.; Spertus, J.A. Perceived Stress in Myocardial Infarction: Long-Term Mortality and Health Status Outcomes. J. Am. Coll. Cardiol. 2012, 60, 1756-1763. [CrossRef]

11. Stewart, R.A.H.; Colquhoun, D.M.; Marschner, S.L.; Kirby, A.C.; Simes, J.; Nestel, P.J.; Glozier, N.; O’Neil, A.; Oldenburg, B.; White, H.D.; et al. Persistent psychological distress and mortality in patients with stable coronary artery disease. Heart 2017, 103, 1860-1866. [CrossRef] [PubMed]

12. Kim, E.S.; Delaney, S.W.; Kubzansky, L.D. Sense of Purpose in Life and Cardiovascular Disease: Underlying Mechanisms and Future Directions. Curr. Cardiol. Rep. 2019, 21, 135. [CrossRef] [PubMed] 